TRANSACTIONS OF THE

AMERICAN MATHEMATICAL SOCIETY

Volume 348, Number 8, August 1996

\title{
FOXBY DUALITY AND GORENSTEIN INJECTIVE AND PROJECTIVE MODULES
}

\author{
EDGAR E. ENOCHS, OVERTOUN M.G. JENDA, AND JINZHONG XU
}

\begin{abstract}
In 1966, Auslander introduced the notion of the $G$-dimension of a finitely generated module over a Cohen-Macaulay noetherian ring and found the basic properties of these dimensions. His results were valid over a local Cohen-Macaulay ring admitting a dualizing module (also see Auslander and Bridger (Mem. Amer. Math. Soc., vol. 94, 1969)). Enochs and Jenda attempted to dualize the notion of $G$-dimensions. It seemed appropriate to call the modules with $G$-dimension 0 Gorenstein projective, so the basic problem was to define Gorenstein injective modules. These were defined in Math. Z. 220 (1995), 611-633 and were shown to have properties predicted by Auslander's results. The way we define Gorenstein injective modules can be dualized, and so we can define Gorenstein projective modules (i.e. modules of $G$-dimension 0) whether the modules are finitely generated or not. The investigation of these modules and also Gorenstein flat modules was continued by Enochs, Jenda, Xu and Torrecillas. However, to get good results it was necessary to take the base ring Gorenstein. H.-B. Foxby introduced a duality between two full subcategories in the category of modules over a local CohenMacaulay ring admitting a dualizing module. He proved that the finitely generated modules in one category are precisely those of finite $G$-dimension. We extend this result to modules which are not necessarily finitely generated and also prove the dual result, i.e. we characterize the modules in the other class defined by Foxby. The basic result of this paper is that the two classes involved in Foxby's duality coincide with the classes of those modules having finite Gorenstein projective and those having finite Gorenstein injective dimensions. We note that this duality then allows us to extend many of our results to the original Auslander setting.
\end{abstract}

\section{INTRODUCTION}

Unless stated otherwise, $R$ will be a local Cohen-Macaulay ring of Krull dimensions $d$ admitting a dualizing module $D$ and with residue field $k$. The Matlis dual of $M$ will be denoted $M^{v}$.

For any $P \in \operatorname{Spec}(R)$, we note that $D_{P}$ is a dualizing module for $R_{P}$. Also, $\hat{D}$ is dualizing for $\hat{R}$ (the completion of $R$ ) and $D / I D$ is dualizing for $R / I$ if $I$ is generated by an $R$-sequence. If the sequence is maximal, then $\operatorname{dim} R / I=0$, so $D / I D \cong E_{R / I}(k)$.

Since $D_{P} \neq 0$ for all $P \in \operatorname{Spec}(R), \operatorname{Hom}(D, M)=0$ implies $M=0$. So then $D \otimes N=0$ implies $N=0$ since $(D \otimes N)^{v}=\operatorname{Hom}\left(D, N^{v}\right)$.

Received by the editors September 7, 1994 and, in revised form, October 2, 1995.

1991 Mathematics Subject Classification. Primary 13C10, 13C11; Secondary 13C99.

(C)1996 American Mathematical Society 
A module $M$ is said to be Gorenstein projective if there is an exact sequence

$$
\cdots \rightarrow P_{2} \stackrel{\partial_{2}}{\rightarrow} P_{1} \stackrel{\partial_{1}}{\rightarrow} P_{0} \stackrel{\partial_{0}}{\rightarrow} P_{-1} \stackrel{\partial_{-1}}{\rightarrow} P_{-2} \rightarrow \cdots
$$

of projective modules such that $M=\operatorname{Ker}\left(\partial_{0}\right)$ and such that $\operatorname{Hom}(-, P)$ leaves the sequence exact whenever $P$ is a projective module. Dually, a module $N$ is said to be Gorenstein injective if there is an exact sequence

$$
\cdots \rightarrow E^{-2} \stackrel{\partial^{-2}}{\rightarrow} E^{-1} \stackrel{\partial^{-1}}{\rightarrow} E^{0} \stackrel{\partial^{0}}{\rightarrow} E^{1} \stackrel{\partial^{1}}{\rightarrow} E^{2} \rightarrow \cdots
$$

of injective modules such that $N=\operatorname{Ker}\left(\partial^{0}\right)$ and such that $\operatorname{Hom}(E,-)$ leaves the sequence exact when $E$ is injective.

If $M$ is Gorenstein projective, $\operatorname{Ext}^{i}(M, L)=0$ for all $i \geq 1$ and $L$ such that proj. $\operatorname{dim} L<\infty$. If $N$ is Gorenstein injective, $\operatorname{Ext}^{i}(L, N)=0$ for $i \geq 1$ and $L$ such that inj. $\operatorname{dim} L<\infty$.

Given a class $\mathcal{F}$ of $R$-modules, a linear map $\phi: F \rightarrow M$ where $F \in \mathcal{F}$ is called an $\mathcal{F}$-precover of $M$ if $\operatorname{Hom}(G, F) \rightarrow \operatorname{Hom}(G, M) \rightarrow 0$ is exact for all $G \in \mathcal{F}$. If, moreover, $f \circ \phi=\phi$ for $f \in \operatorname{Hom}(F, F)$ implies $f$ is an automorphism of $f$, then $\phi$ is called an $\mathcal{F}$-cover of $M$. If an $\mathcal{F}$-cover exists, it is unique up to isomorphism. $\mathcal{F}$ is said to be precovering (covering) if every module admits an $\mathcal{F}$-precover (an $\mathcal{F}$-cover). If $\mathcal{F}$ is precovering, for every $M$ there is a complex

$$
\cdots \rightarrow F_{2} \rightarrow F_{1} \rightarrow F_{0} \rightarrow M \rightarrow 0
$$

with each $F_{i} \in \mathcal{F}$ such that for all $F \in \mathcal{F}$, $\operatorname{Hom}(F,-)$ makes the complex exact. Such a complex is called an $\mathcal{F}$-projective resolution of $M$ and is unique up to homotopy. If $0 \rightarrow M^{\prime} \rightarrow M \rightarrow M^{\prime \prime} \rightarrow 0$ is a complex of modules which becomes exact when Hom: $(F,-)$ is applied to it for all $F \in \mathcal{F}$, then if $M^{\prime}$ and $M^{\prime \prime}$ admit $\mathcal{F}$-projective resolutions, so does $M$, and the resolution of $M$ can be constructed from the resolutions of $M^{\prime}$ and $M^{\prime \prime}$ as usual.

$\mathcal{F}$-preenvelopes and envelopes are defined dual to $\mathcal{F}$-precover and covers. We then have the obvious notions of $\mathcal{F}$-injective resolutions and of $\mathcal{F}$ being preenveloping. $\mathcal{F}$-injective resolutions of $N^{\prime}$ and $N^{\prime \prime}$ can be combined to give one of $N$ if $0 \rightarrow N^{\prime} \rightarrow N \rightarrow N^{\prime \prime} \rightarrow 0$ is a complex such that $\operatorname{Hom}(-, F)$ makes the complex exact whenever $F \in \mathcal{F}$. For example, if $\mathcal{F}$ is the class of injective modules, then the condition on $0 \rightarrow N^{\prime} \rightarrow N \rightarrow N^{\prime \prime} \rightarrow 0$ is just that the sequence is exact.

If $\mathcal{F}$ is, for example, the class of flat modules, then an $\mathcal{F}$-cover is called a flat cover. In this paper we will follow this general convention for terminology.

We recall that by ([6], Proposition 5.1) every module over a noetherian ring $R$ has a flat preenvelope.

Definition. For any $R$, let $\mathcal{L}(R)$ be the class of modules $L$ such that proj. $\operatorname{dim} L$ $<\infty$.

We need the following result.

Proposition 1.1. If $R$ is any local ring then the class $\mathcal{L}=\mathcal{L}(R)$ of modules of finite projective dimension is preenveloping.

Proof. We recall that if proj. $\operatorname{dim} L<\infty$ then proj. $\operatorname{dim}: L \leq \operatorname{dim}: R$ (Raynaud, Gruson [19], p. 84). Then by Jensen ([18]) every flat module has projective dimension at most $\operatorname{dim}: R$. These two results imply that $\mathcal{L}$ is closed under taking products.

Now given any module $M$, it is not hard to see that there is an infinite cardinal $N_{\beta}$ (depending on $M$ ) such that if $\phi: M \rightarrow L$ is linear with $L \in \mathcal{L}$, then there is 
an $L^{\prime} \in \mathcal{L}$ with $\phi(M) \subset L^{\prime} \subset L$ and such that $\operatorname{Card}\left(L^{\prime}\right) \leq N_{\beta}$. But it is also easy to see then that we can construct an $\mathcal{L}$-preenvelope of the form $M \rightarrow \Pi: L_{i}(i \in I)$ of $M$ where $\operatorname{Card}\left(L_{i}\right) \leq N_{\beta}$ for each $i \in I$.

We note that, in general, an $\mathcal{L}$-preenvelope $M \rightarrow L$ need not be injective.

For any $R$, Foxby [16] defines the class $\mathcal{G}_{0}(R)$ to be those $R$-modules $M$ such that $\operatorname{Tor}_{i}(D, M)=0$ and $\operatorname{Ext}_{i}(D, D \otimes M)=0$ for all $i \geq 1$ and such that the natural map $M \rightarrow \operatorname{Hom}(D, D \otimes M)$ is an isomorphism. The class $\mathcal{J}_{0}(R)$ consists of those $N$ such that $\operatorname{Ext}^{i}(D, N)=0$ and $\operatorname{Tor}_{i}(D, \operatorname{Hom}(D, N))=0$ for all $i \geq 1$ and such that $D \otimes \operatorname{Hom}(D, N) \rightarrow N$ is an isomorphism.

Note that if $P$ is projective, then $P \in \mathcal{G}_{0}(R)$. Indeed, $\operatorname{Ext}^{i}(D, D)=0$ and so $\operatorname{Ext}^{i}(D, D \otimes P)=0$ for $i \geq 1$. Also $P \rightarrow \operatorname{Hom}(D, D \otimes P)$ is an isomorphism when $P=R$ and so is an isomorphism for any projective $P$. The functor $D \otimes-$ from $\mathcal{G}_{0}(R)$ to $\mathcal{J}_{0}(R)$ gives an equivalence between these two categories. Similarly $\operatorname{Hom}(D,-): \mathcal{J}_{0}(R) \rightarrow \mathcal{G}_{0}(R)$ is an equivalence. It follows, for example, that

$$
\operatorname{Hom}\left(M_{1}, M_{2}\right) \cong \operatorname{Hom}\left(D \otimes M_{1}, D \otimes M_{2}\right)
$$

for all $M_{1}, M_{2} \in \mathcal{G}_{0}(R)$. We will need

Proposition 1.2 (Foxby, [13, Proposition 1.2]). If $E$ is injective then $E \in \mathcal{J}_{0}(R)$.

Proposition 1.3. If $M$ is Gorenstein projective then $M \in \mathcal{G}_{0}(R)$.

Proof. Let $\cdots P_{1} \stackrel{\partial_{1}}{\rightarrow} P_{0} \stackrel{\partial_{0}}{\rightarrow} P_{-1} \rightarrow \cdots$ be an exact sequence of projective modules with $M=\operatorname{Ker}\left(\partial_{0}\right)$ which is left exact by each $\operatorname{Hom}(-, P)$ when $P$ is projective. If $E$ is injective then $\operatorname{Hom}(D, E)$ has finite flat dimension and hence finite projective dimension. But then $\operatorname{Hom}(-, \operatorname{Hom}(D, E))$ leaves the sequence above exact. Hence $\operatorname{Hom}(D \otimes-, E)$ does too. Since $E$ was arbitrary, $D \otimes-$ leaves the sequence exact. This implies $\operatorname{Tor}_{i}(D, M)=0$ for $i \geq 1$. But also $0 \rightarrow D \otimes M \rightarrow D \otimes P_{0} \rightarrow D \otimes P_{-1}$ is exact, and so

$$
0 \rightarrow \operatorname{Hom}(D, D \otimes M) \rightarrow \operatorname{Hom}\left(D, D \otimes P_{0}\right) \rightarrow \operatorname{Hom}\left(D, D \otimes P_{-1}\right)
$$

is exact. But $P_{i} \cong \operatorname{Hom}\left(D, D \otimes P_{i}\right)$ for each $i$, since each $P_{i}$ is projective and so in $\mathcal{G}_{0}(R)$. But then $D \rightarrow \operatorname{Hom}(D, D \otimes M)$ is an isomorphism. Now let $0 \rightarrow M \rightarrow$ $P_{0} \rightarrow N \rightarrow 0$ be exact. $N$ is also Gorenstein projective, so $N \rightarrow \operatorname{Hom}(D, D \otimes N)$ is an isomorphism. Then $0 \rightarrow D \otimes M \rightarrow D \otimes P_{0} \rightarrow D \otimes N \rightarrow 0$ is exact. Applying $\operatorname{Hom}(D,-)$, we get an exact sequence

$$
0 \rightarrow M \rightarrow P \rightarrow N \rightarrow \operatorname{Ext}^{1}(D, D \otimes M) \rightarrow 0=\operatorname{Ext}^{1}(D, D \otimes P)
$$

(since $\left.\operatorname{Ext}^{1}(D, D)=0\right)$. This implies that $\operatorname{Ext}^{1}(D, D \otimes M)=0$. But then since we also have $\operatorname{Ext}^{1}(D, D \otimes N)=0$ and $\operatorname{Ext}^{2}\left(D, D \otimes P_{0}\right)=0$, we get $\operatorname{Ext}^{2}(D, D \otimes M)=0$. Then by induction we get $\operatorname{Ext}^{i}(D, D \otimes M)=0$ for all $i \geq 1$.

Similar arguments give

Proposition 1.4. If $N$ is Gorenstein injective then $N \in \mathcal{J}_{0}(R)$.

We now let $\mathcal{W}$ be the class of all modules $W$ such that $W \cong D \otimes P$ for some projective module. $\mathcal{U}$ will consist of all $V$ such that $V \cong \operatorname{Hom}(D, E)$ with $E$ an injective module.

Proposition 1.5. $\mathcal{W}$ is a precovering class and $\mathcal{U}$ is a preenveloping class. 
Proof. For any $M$, the evaluation map $D^{(\operatorname{Hom}(D, M))} \rightarrow M$ can be seen to be a $\mathcal{W}$-precover of $M$. Note that $W \rightarrow M$ with $W \in \mathcal{W}$ is a $\mathcal{W}$-precover if and only if $\operatorname{Hom}(D, W) \rightarrow \operatorname{Hom}(D, M) \rightarrow 0$ is exact.

To get a $\mathcal{U}$-preenvelope of $M$, let $D \otimes M \subset E$ with $E$ injective. Then $M \rightarrow$ $\operatorname{Hom}(D, D \otimes M) \rightarrow \operatorname{Hom}(D, E)$ is the desired preenvelope. For given $M \rightarrow$ $\operatorname{Hom}(D, \bar{E})$ with $\bar{E}$ injective, we get a map $D \otimes M \rightarrow D \otimes \operatorname{Hom}(D, \bar{E}) \cong \bar{E}$ (since $\left.\bar{E} \in \mathcal{J}_{0}(R)\right)$. This map can be extended to a map $E \rightarrow \bar{E}$ which in turn gives a map $\operatorname{Hom}(D, E) \rightarrow \operatorname{Hom}(D, \bar{E})$. It can then be checked that the composition $M \rightarrow \operatorname{Hom}(D, E) \rightarrow \operatorname{Hom}(D, \bar{E})$ is the original map $M \rightarrow \operatorname{Hom}(D, \bar{E})$.

Theorem 1.6 (Foxby [13, Lemma 1.3]). Let $0 \rightarrow M^{\prime} \rightarrow M \rightarrow M^{\prime \prime} \rightarrow 0$ be an exact sequence of $R$-modules. Then if any two of $M^{\prime}, M, M^{\prime \prime}$ are in $\mathcal{G}_{0}(R)\left(\mathcal{J}_{0}(R)\right)$, so is the third.

\section{The MAIN THEOREMS}

Theorem 2.1. If $M \in \mathcal{G}_{0}(R)$ and $0 \rightarrow C \rightarrow P_{d-1} \rightarrow \cdots \rightarrow P_{0} \rightarrow M \rightarrow 0$ is exact with $P_{0}, \ldots, P_{d-1}$ projective, then $C$ is Gorenstein projective.

Proof. Let

$$
\cdots \rightarrow P_{d+1} \rightarrow P_{d} \rightarrow P_{d-1} \rightarrow \cdots \rightarrow P_{0} \rightarrow M \rightarrow 0
$$

be a complete projective resolution of $M$. Then since $M \in \mathcal{G}_{0}(R)$,

$$
\cdots \rightarrow D \otimes P_{d+1} \rightarrow D \otimes P_{d} \rightarrow D \otimes P_{d-1} \rightarrow \cdots \rightarrow D \otimes P_{0} \rightarrow D \otimes M \rightarrow 0
$$

is exact. Since $\operatorname{Ext}^{i}(D, D)=0$ for $i \geq 1, \operatorname{Ext}^{i}(D \otimes P, D \otimes Q)=0$ if $P$ and $Q$ are projective and $i \geq 1$. But also inj. $\operatorname{dim} D=d$, so inj. $\operatorname{dim}(D \otimes P) \leq d$ when $P$ is projective. As a consequence, when $P$ is projective and $\operatorname{Hom}(-, D \otimes P)$ is applied to the complex above, the complex becomes exact beginning with the term $\operatorname{Hom}\left(D \otimes P_{d}, D \otimes P\right)$. But for each $i$,

$$
\operatorname{Hom}\left(D \otimes P_{i}, D \otimes P\right) \cong \operatorname{Hom}\left(P_{i}, P\right),
$$

so

$$
\operatorname{Hom}\left(P_{d}, P\right) \rightarrow \operatorname{Hom}\left(P_{d+1}, P\right) \rightarrow \cdots
$$

is exact. This implies $\operatorname{Ext}^{d+i}(M, P)=0$ for $i \geq 1$, and so $\operatorname{Ext}^{i}(C, P)=0$ for $i \geq 1$. This implies $\operatorname{Ext}^{i}(C, L)=0$ if proj. $\operatorname{dim} L<\infty$ and $i \geq 1$. Now by Proposition 1.1, $C$ has an $\mathcal{L}(R)$-preenvelope, $C \rightarrow L$. Since $C \subset P_{d-1}, C \rightarrow L$ is an injection. Let $Q \rightarrow L$ be surjective and linear with $Q$ projective. If $K$ is the kernel of $Q \rightarrow L$, then $K \in \mathcal{L}(R)$, so $\operatorname{Ext}^{1}(C, K)=0$. Hence $C \rightarrow L$ has a lifting $C \rightarrow Q$ which is an injection and still an $\mathcal{L}(R)$-preenvelope. Let $0 \rightarrow C \rightarrow Q \rightarrow B \rightarrow 0$ be exact. Then $\operatorname{Ext}^{i}(B, P)=0$ for $i \geq 1$ and $P$ projective. We now argue that $B$ can be embedded in a module of finite projective dimension. By Theorem 1.6, $B \in \mathcal{G}_{0}(R)$. Let $D \otimes B \subset E$ with $E$ injective. Then $B \cong \operatorname{Hom}(D, D \otimes B) \subset \operatorname{Hom}(D, E)$. By Ishikawa [14], $\operatorname{Hom}(D, E)$ has finite flat and hence finite projective dimension. But then we use the same argument as above and have an embedding $B \subset Q^{1}$ with $Q^{1}$ projective and $B \rightarrow Q^{1}$ an $\mathcal{L}(R)$-preenvelope. Let $Q^{0}=Q$. So $0 \rightarrow C \rightarrow Q^{0} \rightarrow Q^{1}$ is exact. Then proceeding in this manner we can construct an exact sequence $0 \rightarrow C \rightarrow Q^{0} \rightarrow Q^{1} \rightarrow Q^{2} \rightarrow \cdots$ with each $Q^{i}$ projective and such that $\operatorname{Hom}(-, P)$ leaves the sequence exact when $P$ is projective. If

$$
\cdots \rightarrow Q^{-2} \rightarrow Q^{-1} \rightarrow C \rightarrow 0
$$


is any projective resolution of $C$, then since $\operatorname{Ext}^{i}(C, P)=0$ for $i \geq 1$ and $P$ projective, we get the complex

$$
\cdots \rightarrow Q^{-2} \rightarrow Q^{-1} \rightarrow Q^{0} \rightarrow Q^{1} \rightarrow Q^{2} \rightarrow \cdots
$$

having the necessary properties to guarantee that $C$ is Gorenstein projective.

Theorem 2.2. A module $C$ is Gorenstein projective if and only if $C \in \mathcal{G}_{0}(R)$ and $\operatorname{Ext}^{i}(C, L)=0$ for all $i \geq 1$ and all $L$ such that proj. $\operatorname{dim} L<\infty$.

Proof. We only need apply the arguments we used concerning the $C$ in the proof of Theorem 2.1.

Corollary 2.3. If $C=C_{1} \oplus C_{2}$, then $C$ is Gorenstein projective if and only if $C_{1}$ and $C_{2}$ are.

Proof. Immediate from the above.

Remark. If a module $C$ has finite projective dimension and $\operatorname{Ext}^{i}(C, P)=0$ for all $i \geq 1$ and all projective modules $P$, then $C$ is projective. Hence any Gorenstein projective module which is of finite projective dimension is projective.

Now let $M$ be any module of finite projective dimension. Then by Theorem 1.6 we have $M \in \mathcal{G}_{0}(R)$ since $P \in \mathcal{G}_{0}(R)$ for every projective module $P$. If we let $C$ then be as in Theorem 2.2, then by the above $C$ is projective.

Hence proj. $\operatorname{dim} M \leq d$. This gives another proof of Raynaud and Gruson's result (see [19], p. 84) that proj. $\operatorname{dim} M \leq \operatorname{dim} R$ whenever proj. $\operatorname{dim} M<\infty$, but of course, under more restrictive conditions.

Corollary 2.4. $M \in \mathcal{G}_{0}(R)$ if and only if for some $n \geq 0$ there is an exact sequence

$$
0 \rightarrow C_{n} \rightarrow \cdots \rightarrow C_{0} \rightarrow M \rightarrow 0
$$

with $C_{0}, \ldots, C_{n}$ Gorenstein projective modules. If there is such an exact sequence, then there is one with $n \leq d$.

Proof. By Theorem 1.6, Proposition 1.3 and Theorem 2.1.

See Foxby (Theorem 1 of [15]) for the corresponding result when $M$ is finitely generated. Similarly we have

Theorem 2.5. If $N \in \mathcal{J}_{0}(R)$ and

$$
0 \rightarrow N \rightarrow E^{0} \rightarrow \cdots \rightarrow E^{d-1} \rightarrow G \rightarrow 0
$$

is exact, where $E^{0}, \ldots, E^{d-1}$ are injective, then $G$ is Gorenstein injective.

Corollary 2.6. $N \in \mathcal{J}_{0}(R)$ if and only if for some $n \geq 0$ there exists an exact sequence

$$
0 \rightarrow N \rightarrow G^{0} \rightarrow \cdots \rightarrow G^{n} \rightarrow 0
$$

with $G^{0}, \ldots, G^{n}$ Gorenstein injective. In this case there exists such a sequence with $n \leq d$.

Similar arguments gives the next two results.

Theorem 2.7. A module $G$ is Gorenstein injection if and only if $G \in \mathcal{J}_{0}(R)$ and $\operatorname{Ext}^{i}(L, G)=0$ for all $i \geq 1$ and all $L$ such that $\operatorname{inj} . \operatorname{dim} L<\infty$.

Corollary 2.8. If $G=G_{1} \oplus G_{2}$, then $G$ is Gorenstein injective if and only if $G_{1}$ and $G_{2}$ are. 
Remark. We can also deduce now that if inj. $\operatorname{dim} N<\infty$ for any $N$, then inj. $\operatorname{dim} N$ $\leq d$

We can now prove

Theorem 2.9. If $M \in \mathcal{G}_{0}(R)$, then $M$ has a Gorenstein projective precover $C \rightarrow$ $M$ whose kernel belongs to $\mathcal{L}(R)$.

Proof. Let $0 \rightarrow D \rightarrow P_{d-1} \rightarrow \cdots \rightarrow P_{0} \rightarrow M \rightarrow 0$ be exact with $P_{0}, \ldots, P_{d-1}$ projective. Then by Theorem 2.1, $D$ is Gorenstein projective. Let

$$
\cdots \rightarrow Q^{-1} \stackrel{\partial^{-1}}{\rightarrow} Q^{0} \stackrel{\partial^{0}}{\rightarrow} Q^{1} \stackrel{\partial^{1}}{\rightarrow} Q^{2} \rightarrow \cdots
$$

be an exact sequence of projective modules with $\operatorname{Ker}\left(\partial^{0}\right)=D$ and such that $\operatorname{Hom}(-, P)$ leaves the sequence exact when $P$ is projective. Let $0 \rightarrow D \rightarrow Q^{0} \rightarrow$ $\cdots \rightarrow Q^{d-1} \rightarrow C \rightarrow 0$ be exact. Then our hypothesis guarantees that there is a commutative diagram

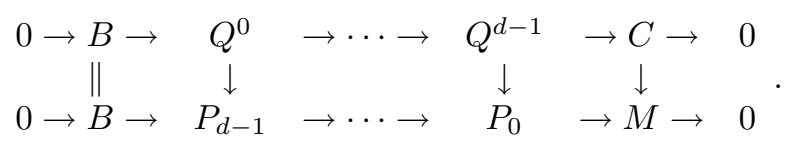

Form the complex associated to this diagram thought of as a double complex (with 0 in all other positions), namely

$$
0 \rightarrow D \rightarrow D \oplus Q^{0} \rightarrow \cdots \rightarrow P_{0} \oplus C \rightarrow M \rightarrow 0 .
$$

This complex is exact since the rows of the diagram above are exact. This complex has the exact sequence $0 \rightarrow D \rightarrow D \rightarrow 0$ as a subcomplex, so we get an exact quotient complex $0 \rightarrow Q^{0} \rightarrow \cdots \rightarrow P_{1} \oplus Q^{d-1} \rightarrow P_{0} \oplus C \rightarrow M \rightarrow 0$. Since all terms of this sequence with (possibly) the exceptions of $P_{0} \oplus C$ and $M$ are projective, we see that if

$$
0 \rightarrow L \rightarrow P_{0} \oplus C \rightarrow M \rightarrow 0
$$

is exact, then proj. $\operatorname{dim} L<\infty$. In fact, for future reference, we note that proj. $\operatorname{dim} L$ $\leq d-1$. Both $P_{0}$ and $C$ are Gorenstein projective, so $P_{0} \oplus C$ is too. Since $\operatorname{Ext}^{1}(D, L)=0$ for all Gorenstein projective modules $D, P_{0} \oplus C \rightarrow M$ is a Gorenstein projective precover.

A dual argument gives

Theorem 2.10. If $N \in \mathcal{J}_{0}(R)$, then $N$ has a Gorenstein injective preenvelope $N \subset G$ such that inj. $\operatorname{dim} G / N \leq d-1$.

We note that Auslander and Buchweitz [3] have results similar to Theorems 2.9 and 2.10 with chain conditions on the modules.

The proof Theorem 6.1 of [12] can be modified to show that in fact every $N \in \mathcal{J}_{0}(R)$ has a Gorenstein injective envelope $N \subset G$. It will follow then that inj. $\operatorname{dim} G / N \leq d-1$ still holds.

\section{Gorenstein flat modules AND COVERS}

We recall some facts concerning flat covers. First we note that any module of finite flat dimension has a flat cover (Theorem 3.2, [4]). If $F \rightarrow M$ is a flat cover of $M$ with kernel $K$, then $K$ is cotorsion (Lemma 2.2, [5]), i.e. $\operatorname{Ext}^{1}(G, K)=0$ whenever $\mathrm{G}$ is flat. Hence if $M$ is itself cotorsion, then so is $F$. If a module $K$ is flat and cotorsion, then $K$ is a summand of a module of the form $\operatorname{Hom}(E, \bar{E})$ where $E$ and $\bar{E}$ are injective (Lemma 2.3, [5]). 
An $R$-module $X$ is called Gorenstein flat if there is an exact sequence

$$
\cdots \rightarrow F^{-2} \rightarrow F^{-1} \rightarrow F^{0} \stackrel{\partial^{0}}{\rightarrow}: F^{1} \rightarrow F^{2} \rightarrow \cdots
$$

with $F^{i}$ flat and such that $X=\operatorname{Im}\left(\partial^{0}\right)$ and such that $E \otimes-$ leaves the sequence exact whenever $E$ is an injective module.

Gorenstein flat modules were introduced in [11]. In [20], it was shown that every module over a Gorenstein ring has a Gorenstein flat cover. In this section we will show that every element in the class $\mathcal{G}_{0}(R)$ has a Gorenstein flat cover. Below we argue that the class of Gorenstein flat modules is closed under taking direct limits, hence by (Theorem, p. 207, [6] or [12]) it will suffice to show that every module in $\mathcal{G}_{0}(R)$ has a Gorenstein flat precover. If $M \in \mathcal{G}_{0}$ and $0 \rightarrow X \rightarrow Y \rightarrow M \rightarrow 0$ is exact with $Y$ Gorenstein flat and $X$ such that $\operatorname{Ext}^{1}(Z, X)=0$ whenever $Z$ is Gorenstein flat, then $Y \rightarrow M$ is a Gorenstein flat precover of $M$; hence we will produce such an exact sequence.

We need

Lemma 3.1. If the sequence

$$
\cdots \rightarrow F^{-1} \rightarrow F^{0} \rightarrow F^{1} \rightarrow \cdots
$$

is an exact sequence of flat modules such that $E \otimes-$ leaves the sequence exact when $E$ is an injective module and $K$ is cotorsion and of finite flat dimesion, then $\operatorname{Hom}(-, K)$ leaves the sequence exact.

Proof. If $K$ is flat and cotorsion, then by the remarks above, $\operatorname{Hom}(-, K)$ will leave the sequence exact if $\operatorname{Hom}(-, \operatorname{Hom}(E, \bar{E}))$ leaves the sequence exact when $E$ and $\overline{\mathrm{E}}$ are injective. But $\operatorname{Hom}(-, \operatorname{Hom}(E, \bar{E}))$ and $\operatorname{Hom}(E \otimes-, \bar{E})$ are isomorphic functors. So for such a $K, \operatorname{Hom}(-, K)$ leaves the sequence exact.

Now suppose $0 \rightarrow K^{\prime} \rightarrow K \rightarrow K^{\prime \prime} \rightarrow 0$ is an exact sequence of modules such that $\operatorname{Hom}(F,-)$ leaves this sequence exact whenever $F$ is flat. Then applying each of $\operatorname{Hom}\left(-, K^{\prime}\right), \operatorname{Hom}(-, K)$ and $\operatorname{Hom}\left(-, K^{\prime \prime}\right)$ to the sequence

$$
\cdots \rightarrow F^{-1} \rightarrow F^{0} \rightarrow F^{1} \rightarrow \cdots,
$$

we get a short exact sequence of complexes. Hence if any two of $\operatorname{Hom}\left(-, K^{\prime}\right)$, $\operatorname{Hom}(-, K)$ and $\operatorname{Hom}\left(-, K^{\prime \prime}\right)$ leave the given sequence exact, so does the third. Now we note that by induction, we can generalize this result to exact sequences with any finite number of terms. Now let $K$ be cotorsion and of finite flat dimension. Then using flat covers we construct an exact sequence

$$
0 \rightarrow F_{n} \rightarrow \cdots \rightarrow F_{0} \rightarrow K \rightarrow 0
$$

with each $F_{0}, \cdots, F_{n}$ flat and cotorsion and such that $\operatorname{Hom}(F,-)$ leaves the sequence exact whenever $F$ is flat. But by the above, $\operatorname{Hom}\left(-, F_{i}\right)$ leaves the sequence

$$
\cdots \rightarrow F^{-1} \rightarrow F^{0} \rightarrow F^{1} \rightarrow \cdots
$$

exact for each $i=0, \cdots, n$. Hence $\operatorname{Hom}(-, K)$ also leaves the sequence exact.

Corollary 3.2. If $X$ is Gorenstein flat and $K$ is cotorsion and of finite flat dimension, then $\operatorname{Ext}^{i}(X, K)=0$ for $i \geq 1$.

Theorem 3.3. Let $X$ be an $R$-module. Then the following are equivalent.

(1) $X$ is Gorenstein flat.

(2) $X \in \mathcal{G}_{0}$ and $\operatorname{Tor}_{i}^{R}(L, X)=0$ for all modules $L$ of finite injective dimension and $i>0$. 
Proof. (1) $\Longrightarrow(2)$. Since $X$ is Gorenstein flat, by the definition there is an exact sequence

$$
\cdots \rightarrow F^{-2} \rightarrow F^{-1} \rightarrow F^{0} \stackrel{\partial^{0}}{\rightarrow}: F^{1} \rightarrow F^{2} \rightarrow \cdots
$$

with $F^{i}$ flat such that $X=\operatorname{Im}\left(\partial^{0}\right)$ and $E \otimes-$ leaves the sequence exact for any injective module $E$. Easily for any module $L$ of finite injective dimension, $L \otimes-$ leaves the sequence exact. Note that $X=\operatorname{Im}\left(\partial^{0}\right)$. It follows that $\operatorname{Tor}_{i}^{R}(L, X)=0$ for all $i>0$ and $L$ of finite injective dimension. In particular, $\operatorname{Tor}_{i}^{R}(D, X)=0$ for all $i>0$. Then using the left exactness of $\operatorname{Hom}(D,-)$ and the fact that $F^{i} \rightarrow$ $\operatorname{Hom}\left(D, D \otimes F^{i}\right)$ is an isomorphism for ever $i \geq 0$, we have a commutative diagram with exact rows

$$
\begin{array}{rlclll}
0 \rightarrow & X & F^{0} & \rightarrow & \\
\downarrow & & \downarrow f_{0} & & \\
0 \rightarrow \operatorname{Hom}(D, D \otimes X) & \rightarrow & \operatorname{Hom}\left(D, D \otimes F^{0}\right) & \rightarrow & \\
& \rightarrow & F^{1} & \rightarrow & F^{2} \\
& & \downarrow f_{1} & & \downarrow f_{2} \\
& & \rightarrow & \operatorname{Hom}\left(D, D \otimes F^{1}\right) & \rightarrow & \operatorname{Hom}\left(D, D \otimes F^{2}\right)
\end{array}
$$

Note that all flat modules are in the class $\mathcal{G}_{0}$. It follows that $f_{0}, f_{1}$ and $f_{2}$ are all isomorphisms. Hence $X \cong \operatorname{Hom}(D, D \otimes X)$. In order to show that $X$ is in $\mathcal{G}_{0}$, we then only need to show that $\operatorname{Ext}_{R}^{i}(D, D \otimes X)=0$ for all $i>0$.

Consider the exact sequence $0 \rightarrow X \rightarrow F^{0} \rightarrow Y \rightarrow 0$ with $Y=F^{0} / X$. Note that $Y$ is Gorenstein flat. And then by the above, $\operatorname{Tor}_{i}^{R}(D, Y)=0$ and $Y \cong$ $\operatorname{Hom}(D, D \otimes Y)$. Therefore we have the commutative diagram

$$
\begin{aligned}
& 0 \rightarrow \quad X \quad \rightarrow \quad F^{0} \quad \rightarrow \\
& \downarrow \quad \downarrow f_{0} \\
& 0 \rightarrow \operatorname{Hom}(D, D \otimes X) \quad \rightarrow \quad \operatorname{Hom}\left(D, D \otimes F^{0}\right) \quad \rightarrow \\
& \rightarrow \quad Y \quad \rightarrow \quad 0 \\
& \downarrow f_{1} \\
& \rightarrow \operatorname{Hom}(D, D \otimes Y) \rightarrow \operatorname{Ext}^{1}(D, D \otimes X) \rightarrow 0
\end{aligned}
$$

This implies that $\operatorname{Ext}^{1}(D, D \otimes X)=0$. Similarly, $\operatorname{Ext}^{1}(D, D \otimes Y)=0$, and then $\operatorname{Ext}^{2}(D, D \otimes X)=0$.

Repeating the same argument, we easily see that $\operatorname{Ext}^{i}(D, D \otimes X)=0$ for all $i>0$. This finishes the implication $(1) \Rightarrow(2)$.

$(2) \Longrightarrow(1)$. We need to construct a complete flat resolution of $X$ which is left exact by $E \otimes-$ for any injective module $E$.

By assumption, $\operatorname{Tor}_{i}^{R}(E, X)=0$ for any injective module $E$. It follows that for any flat resolution of $X$

$$
\cdots \rightarrow F^{-2} \rightarrow F^{-1} \rightarrow F^{0} \rightarrow X \rightarrow 0
$$


$E \otimes-$ leaves the sequence exact. Then we only need to find the right half of the desired complete flat resolution of $X$.

Set $Y=D \otimes X$. By hypothesis, $\operatorname{Ext}^{i}(D, Y)=0$ for $i>0$. Let $0 \rightarrow Y \rightarrow$ $E \rightarrow Z \rightarrow 0$ be an exact sequence with $E$ the injective envelope of $Y$. Since $\operatorname{Ext}^{1}(D, Y)=0$, we have the exact sequence

$$
0 \rightarrow \operatorname{Hom}(D, Y) \rightarrow \operatorname{Hom}(D, E) \rightarrow \operatorname{Hom}(D, Z) \rightarrow 0 .
$$

Note that $X \cong \operatorname{Hom}(D, Y)$ and that $L=\operatorname{Hom}(D, E)$ has finite flat dimension. Let $W=\operatorname{Hom}(D, Z)$. We have the exact sequence $0 \rightarrow X \rightarrow L \rightarrow W \rightarrow 0$.

We know that $L$ has a flat cover (Theorem 3.2, [4]). Consider the exact sequence

$$
0 \rightarrow K \rightarrow F \rightarrow L \rightarrow 0
$$

with $F \rightarrow L$ the flat cover of $L$. Then we consider the pullback diagram

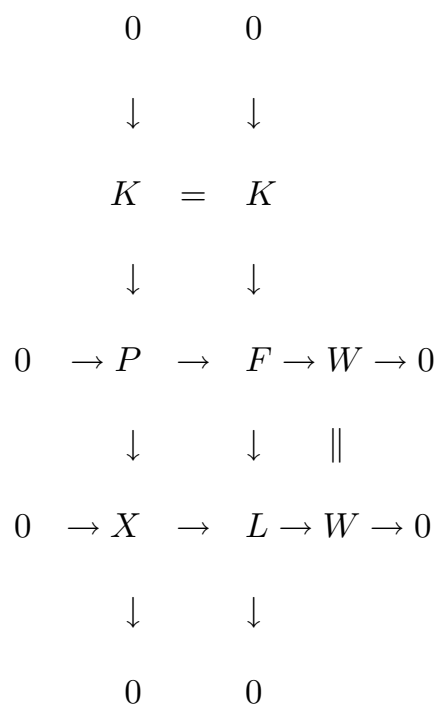

The fact that $\operatorname{Ext}^{1}(X, K)=0$ shows that $0 \rightarrow K \rightarrow D \rightarrow X \rightarrow 0$ is split. Hence $X$ can be embedded into a flat module $F$.

Next since $R$ is noetherian, we can choose an embedding $F$ of $X$ such that $X \rightarrow F$ is a flat preenvelope of $X$, that is, we have an exact sequence $0 \rightarrow X \rightarrow$ $F \rightarrow X_{1} \rightarrow 0$. Therefore the induced sequence

$$
0 \rightarrow \operatorname{Hom}\left(X, F^{\prime}\right) \rightarrow \operatorname{Hom}\left(F, F^{\prime}\right) \rightarrow \operatorname{Hom}\left(X_{1}, F^{\prime}\right) \rightarrow 0
$$

is exact for any flat module $F^{\prime}$. In particular, for any injective module $E$, $\operatorname{Hom}(E, E(k))$ is flat, and then $\operatorname{Hom}(-, \operatorname{Hom}(E, E(k)))$ leaves the above sequence exact. This implies that $E \otimes-$ leaves the sequence exact for any injective module E.

By Theorem 1.6, $X_{1}$ is in $\mathcal{G}_{0}$. It is easy to see that $\operatorname{Tor}_{i}\left(L, X_{1}\right)=0$ for any $L$ of finite injective dimension. and $i>0$. Finally, repeating the same argument, we get the desired complete flat resolution of $X$, and then that $X$ is Gorenstein flat.

Corollary 3.4. Any Gorenstein projective module is Gorenstein flat. Any direct limit of a family of Gorenstein flat modules is Gorenstein flat. 
Proof. Let $C$ be a Gorenstein projective module. By Theorem 3.3 and 2.2, we only need to show that $\operatorname{Tor}_{i}(L, C)=0$ for all $i>0$ and all $L$ of finite injective dimension. But by a natural isomorphism we easily have that

$$
\operatorname{Hom}\left(\operatorname{Tor}_{i}(L, C), E(k)\right) \cong \operatorname{Ext}_{i}(C, \operatorname{Hom}(L, E(k)))=0
$$

since $C$ is Gorenstein projective and $\operatorname{Hom}(L, E(k))$ has finite projective dimension.

The remaining part follows from Theorem 3.3 since $\mathcal{G}_{0}$ is closed under direct limits.

Theorem 3.5. Any $R$-module $M$ in $\mathcal{G}_{0}(R)$ admits a Gorenstein flat cover.

Proof. First we note that the class of Gorenstein flat modules is closed under direct limits. So, in order to find a Gorenstein flat cover of $M$, we only need to find a Gorenstein flat precover of $M$ (which means that the condition (1) holds). By Theorem 2.9 there is an exact sequence

$$
0 \rightarrow K \rightarrow P \rightarrow M \rightarrow 0
$$

such that $P$ is Gorenstein projective and $K$ is of finite projective dimension. Now we claim that there exists an exact sequence $0 \rightarrow K \rightarrow X \rightarrow F \rightarrow 0$ with $F$ flat and $\operatorname{Ext}^{i}\left(F^{\prime}, X\right)=0$ for all Gorenstein flat modules $F^{\prime}$ and $i>0$.

Since $K$ has finite flat dimension, it has flat cover. So we have an exact sequence $0 \rightarrow \bar{K} \rightarrow G \rightarrow K \rightarrow 0$ with $G$ the flat cover of $K$ and the kernel $\bar{K}$ cotorsion. Note that $\bar{K}$ has finite flat dimension. By Corollary $3.2, \operatorname{Ext}^{i}\left(F^{\prime}, \bar{K}\right)=0$ for all Gorenstein flat modules $F^{\prime}$ and $i>0$. Now we consider the pushout diagram

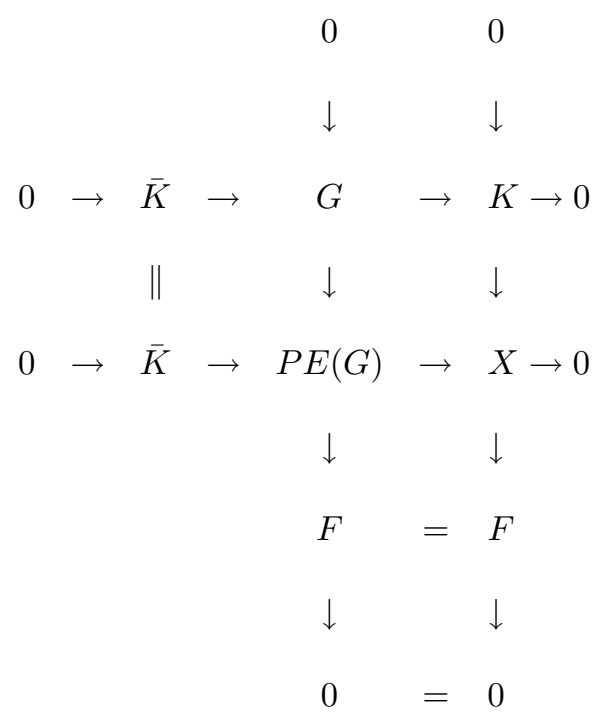

where $P E(G)$ is the pure injective envelope of $G$. Note that $F$ is flat and $\operatorname{Ext}^{i}\left(F^{\prime}, X\right)$ $=0$ for all Gorenstein flat modules $F^{\prime}$ and $i>0$. 
Finally we consider the following pushout diagram

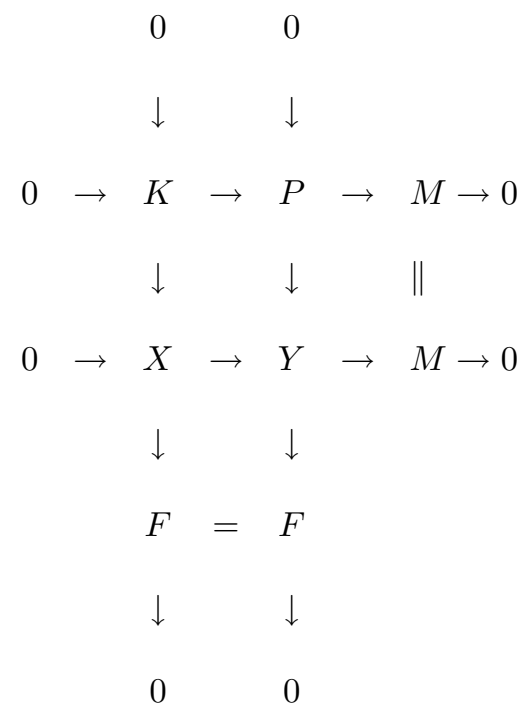

where $Y$ is Gorenstein flat since both $P$ and $F$ are Gorenstein flat. Since $X$ has the property $\operatorname{Ext}^{1}\left(F^{\prime}, X\right)=0$ for all Gorenstein flat modules $F^{\prime}$, it is easy to see that $Y \rightarrow M$ is a Gorenstein flat precover of $M$. Therefore $M$ has a Gorenstein flat cover.

Remark. Let $M \in \mathcal{G}_{0}(G)$, and let $G \rightarrow M$ be a Gorenstein flat cover and $K$ the kernel. By Theorem 2.9 and the above argument, it is easy to see that $K$ has flat dimension less than or equal to $d-1$. The following result indicates how far a Gorenstein flat module differs from a Gorenstein projective module.

Theorem 3.6. If $X$ is Gorenstein flat, then there is an exact sequence $0 \rightarrow F \rightarrow$ $P \rightarrow X \rightarrow 0$ such that $P$ is Gorenstein projective and $F$ is flat.

Proof. Note that a Gorenstein flat module of finite flat dimension is flat. Then the conclusion follows easily from Theorem 2.9 .

\section{REFERENCES}

[1] M. Auslander, Anneaux de Gorenstein et torsion en algèbre commutative, séminaire d'algèbre commutative, Ecole Normale Supérieure de Jeunes Filles, Paris 1966/67. MR 37:1435

[2] M. Auslander and M. Bridger, Stable Module Theory, Memoirs A.M.S. 94, A.M.S., Providence, R.I., 1969. MR 42:4580

[3] M. Auslander and R.O. Buchweitz, Maximal Cohen-Macaulay approximations, Soc. Math. France, Mémoire 38 (1989), 5-37. MR 91h:13010

[4] R. Belshoff, E. Enochs and J. Xu, The existence of flat covers, PAMS 122 (1994), 985-991. MR 95b:16001

[5] E. Enochs, Flat covers and flat cotorsion modules, Proc. AMS 92 (1984), 179-184. MR 85j: 13016

[6] E. Enochs, Injective and flat covers, envelopes and resolvents, Israel J. Math 39 (1981), 33-38. MR 83a:16031

[7] E. Enochs and O.M.G. Jenda, Gorenstein injective and projective modules, Math. Zeit. 220 (1995), 611-633. CMP 1996:5

[8] E. Enochs and O.M.G. Jenda, Gorenstein balance of Hom and Tensor, Tsukuba J. Math. 19 (1995), 1-13. CMP 1995:17 
[9] E. Enochs and O.M.G. Jenda, On Gorenstein injective modules, Comm. Alg. 2 (10) (1993), 3489-3501. MR 94g:13006

[10] E. Enochs and O.M.G. Jenda, Mock finitely generated Gorenstein injective modules and isolated singularities, J. Pure Applied Algebra 96 (1994), 259-269. MR 95i: 13007

[11] E. Enochs, O.M.G. Jenda and B. Torrecillas, Gorenstein flat modules, J. Nanjing University, 10 (1) (1993), 1-9. MR 95a:16004

[12] E. Enochs, O.M.G. Jenda and J. Xu, Covers and envelopes over Gorenstein rings (to appear in Tsukuba J. Math.).

[13] H.-B. Foxby, Gorenstein modules and related modules, Math. Scand. 31 (1972), 267284. MR 48:6094

[14] H.-B. Foxby, Duality homomorphisms for modules over certain Cohen-Macaulay rings, Math. Zeit. 132 (1973), 215-226. MR 48:6095

[15] H.-B. Foxby, Quasi-perfect modules over Cohen-Macaulay rings, Math. Nachr. 66 (1975), 103-110. MR 51:12838

[16] H.-B. Foxby, Gorenstein dimensions over Cohen-Macaulay rings, Proceedings of the international conference on commutative algebra, W. Bruns (editor), Universität Osnabrück, 1994.

[17] T. Ishikawa, On injective and flat modules, J. Math. Soc. Japan 17 (1965), 291-296. MR 32:5711

[18] C.U. Jensen, On the vanishing of $\varliminf^{(i)}$, J. Algebra 15 (1970), 151-169. MR 41:5460

[19] M. Raynaud and L. Gruson, Critères de platitude et de projectivité, Invent. Math. 13 (1971), 1-89. MR 46:7219

[20] J. Xu and E. Enochs, Gorenstein flat covers of modules over Gorenstein rings (to appear in J. Algebra).

(E.E. Enochs and J. Xu) Department of Mathematics, University of Kentucky, LeXINGTON, KENTUCKY 40506-0027

(O.M.G. Jenda) Department of Discrete Mathematics and Statistics, Auburn University, Auburn, Alabama 36849-5307 